\title{
Should colour be considered as a significant design variable in foot orthoses prescription writing?
}

Simon K Spooner ${ }^{1}$ BSc, PhD

Podiatrist

Ian B Griffiths ${ }^{2,3}$ BSc (Hons), MSc, FCPodM FFPM RCPS (Glasg)

Podiatrist

1. Private Practice

Peninsula Podiatry, Plymouth

2. Private Practice

Pure Sports Medicine, London

3. Visiting Lecturer

Centre for Sports \& Exercise Medicine

Queen Mary University of London

\section{Corresponding author}

Simon Spooner

Email: skspooner@blueyonder.co.uk

\section{Author information}

SKS: https://orcid.org/0000-0002-0618-2592

IBG: https://orcid.org//0000-0002-3251-8969

\section{Declaration}

This work did not receive any grant from funding agencies in the public, commercial, or not-for-profit sectors. All authors have no competing interests to declare. Ethical approval was not required. SKS conceived the aim and format of the paper. SKS and IBG made substantial contributions to all drafts of the text.

\section{Key Words}

Foot orthoses, Orthotics, Colour psychology, Podiatry, Biopsychosocial 


\begin{abstract}
Colours act as stimuli capable of inducing physiological and psychological responses, impacting on human life by influencing behaviour, cognition and emotion. Designers frequently draw upon colour association cues in designing products, packaging and marketing materials to exploit the reported human reactions to them. To date, clinicians and foot orthoses manufacturers appear to have given little thought to how the colour of foot orthoses may have impact within the broader context of a biopsychosocial model of healthcare. Here we examine research regarding colour perception and association and provide rationale for how the use of colour in foot orthoses therapy might be better exploited to improve patient outcomes and to form a focus for future research.
\end{abstract}

\title{
Introduction
}

Modern foot orthoses are generally constructed from plastics (e.g. polyethylene or polypropylene) or plastic foams (e.g. ethylene vinyl acetate or polyurethanes). Such materials are available in a wide palette of colours, as well as black and white (figure 1). Manufacturers of medical-grade prefabricated foot orthoses often use colour to distinguish between their products; their orthoses commonly having identical surface geometry but being manufactured from a different density of the same material: each colour denoting a specific durometer of the material. This choice of colour seems arbitrary with no consensus between manufacturers. Moreover, while practitioners are usually asked to specify properties such as type, thickness and durometer of materials when completing foot orthoses laboratory prescription forms for custom devices, rarely (if ever) are they asked which colour they should prefer/require. Here we examine research regarding colour perception and association and provide rationale for how the use of colour in orthoses manufacture might be better exploited to improve patient outcomes with foot orthoses therapy.
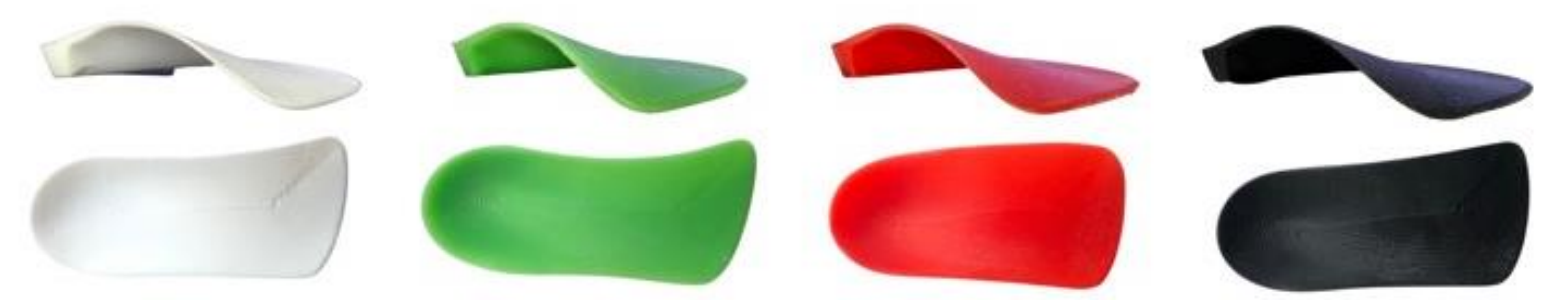
Figure 1: An example of some colours of polypropylene available for use, with almost any colour choice possible when also considering the top cover materials that can be used.

\section{Colour perception and associations}

Colour perception is a subject specific, psychological experience produced by the effect of the reflected light having wavelengths within the visible spectrum, interacting with the nervous system. Colour perception in humans is highly developed and has been the focus of considerable scientific study. Colours act as stimuli capable of inducing physiological and psychological responses, impacting on human life by influencing behaviour, cognition and emotion ${ }^{1}$.

Colour associations appear partly learned and partly ingrained from evolution; they are often societal and context specific. Green and blue are commonly seen as being "natural" with their associations with vegetation and the sky respectively ${ }^{2}$. Fire and the sun with their red and yellow colourations have long provided warmth to the body, but both fire and the sun can also cause injury and pain; so it is little surprise that both yellow and red are colours often used in warning signs to grab the observer's attention. Within the animal kingdom warning colouration (aposematism) has evolved to make the animal, for example a wasp, conspicuous to predators such that it is noticed, remembered and avoided. Aposematism in animals has been shown to influence the emotional response of humans and increase their desire to protect such species $^{3}$.

Designers draw upon colour association cues that are both learned and evolved to exploit the reported human reactions to them. If we take, for example, the use of signage commonly employed on the carriageways and within the workplace in the United Kingdom we find heavy use of red, yellow and black, which are commonly viewed within Western culture as denoting warning and danger signals and draw from aposematism in nature (figure 2). These colours were clearly chosen by design rather than by chance, in order to better ensure increased compliance from the UK population, and their success is likely due (at least in part) to the fact that colour within designs can have a great impact on the behaviour of sighted humans. 

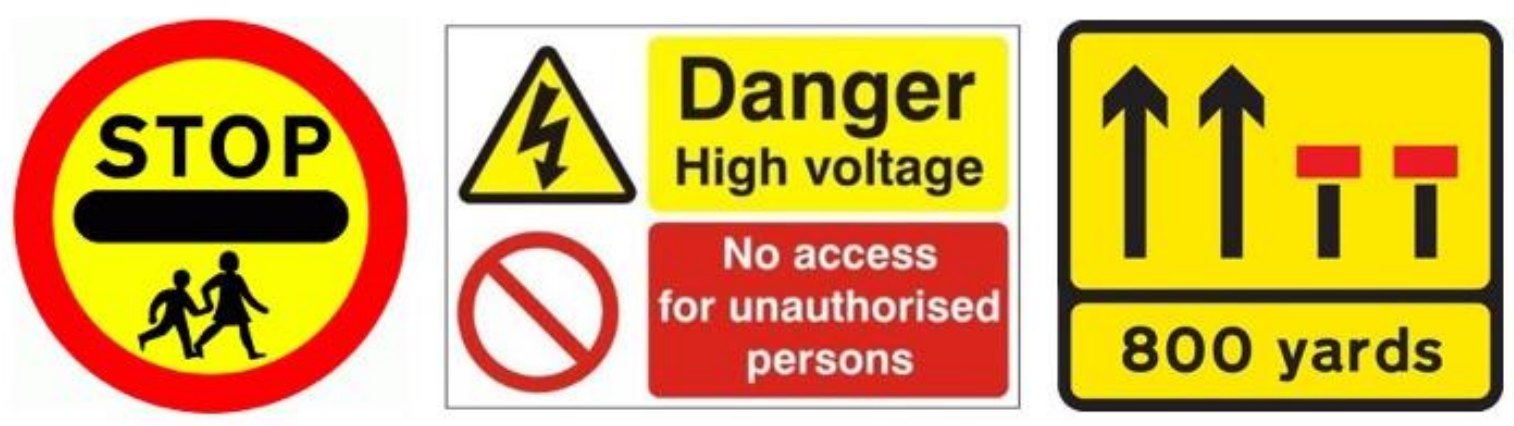

Figure 2: Examples of colour association used in signage within the United Kindgom

\section{Colour and temperature}

Colour association cues are commonly employed by interior and product designers. Taps within kitchens and bathrooms are usually colour coded red for hot and blue for cold; rooms painted in orange and yellow are perceived as being warmer than those painted in colours from the opposite side of the colour-wheel such as blues and mauves. Colour associations with temperature are a form of crossmodal correspondence and are so ingrained that when an incongruent pairing of red with a cold stimulus or blue with a hot stimulus are applied then there is a delay in the response time of the brain to the true stimulus ${ }^{4}$. Perhaps counter-intuitively, when humans are presented with stimuli from two objects of the same temperature, a blue object is more likely to be perceived as being warmer than a red object ${ }^{5}$.

\section{Colour and pain}

Colour association has been utilised to enable patients to describe their pain to clinicians. In a study of patients with osteoarthritis, the majority of subjects chose red to describe their highest intensity pain; associations were made with inflammation, fire and anger. Participants used a range of colours to describe the absence of pain: white, yellow, orange, green and blue, they reported choosing these colours because of their association with positive emotional experiences, for example calmness, purity, happiness or sunshine. Aching pain was consistently identified as black or grey, while sharp intermittent pain was denoted using a range of colours such as red, yellow, purple and white ${ }^{6}$.

Recent research reported on an association between colour and perceived pain intensity. When exposed to identical pain stimuli preceded by a colour cue, the colour red appeared to evoke a higher rated pain intensity when compared to blue or green colour cues and that painful stimuli were rated 
higher following colour cues than when a no colour (black) control cue was employed ${ }^{7}$.

\section{Colour and drugs}

Human responses to colour have been employed in pharmaceutical research and product development. A systematic review concluded that colours effect the perceived action of a drug and appear to influence the effectiveness of the drugs themselves; drugs coloured red, yellow and orange were associated with a stimulant effect while drugs coloured blue and green were associated with having a tranquilising effect ${ }^{8}$. It is worthy of note that although the colour of a pill can only be observed momentarily prior to it being swallowed, it still appears to influence its response post-ingestion.

\section{Colour, athletic performance and biomechanics}

Of all the colours within the visible spectrum, it is the colour red that has received the greatest research attention, particularly in sport. Previous studies have suggested that wearing the colour red during sporting contests has the potential to provide an advantage and the ability to influence the outcome of the contest itself ${ }^{1}$, but this is contentious ${ }^{9}$. While it is beyond the scope of this article to review all of the research related to "the red effect" in sport, the review by Elliot and Maier ${ }^{1}$ provides useful background reading for those with an interest. Instead, we have focused here on the more relevant studies that have examined the relationship between colour and biomechanical variables:

Elliott and Aarts explored the influence of viewing the colour red on the force and velocity of motor outputs ${ }^{10}$. The colour grey was compared to red for the maximal force on a pinch grip test; colour had a significant influence with red yielding higher forces than grey. In a second experiment, the author's compared red with blue and grey in an investigation of maximum force, total force over time and velocity of force development. Maximum force, mean force over time and velocity of force were all significantly higher in association with viewing red than in blue and grey associations, which showed no statistical difference between each other.

In a study of simulated combat it was reported that contestants wearing red outfits showed higher heart rates than those wearing blue jerseys during the fight; there was no significant differences prior to or after the fight. Dynamometry revealed that pre-fight strength was higher in those wearing red compared to blue and higher than in the control condition of subjects wearing their own clothing (neither red nor blue) ${ }^{11}$. 
The effect of the colour of footwear on vertical countermovement jump height performance was investigated in 36 male basketball players. Subjects were tested in three identical shoes differing only in the colour of the upper: red, blue and black. Prior to testing participants indicated their colour preference for the shoes. Participants who preferred the red shoes perceived themselves as being taller when wearing the red shoes compared to the black shoes. Subjects who preferred black shoes had a longer flight time when wearing red shoes compared to the blue shoes ${ }^{12}$.

\section{Colour and foot orthoses research}

Little attention has been given to foot orthoses colour within the scientific literature, indeed only one such study appears to have been published. Wang et al. compared three different insoles in a study of perceived comfort and landing biomechanics in healthy basketball players ${ }^{13}$. Two of the insoles were identical in material and their surface geometry's which included an "arch support", they differed only in the colour of the top-cover; one being white and the other being red. The control insole was a white coloured flat sheet of the same polyurethane material. They reported significant differences in the ankle kinetics when participants wore the red orthoses with arch support, when compared to the white arch support orthoses; the white arch support orthoses did not alter kinetics significantly when compared to the flat control insoles. While there was no difference in the 3D kinematics during the droplandings, the red insoles were perceived by the subjects as being more comfortable. Given that the material and mechanical characteristics of the two arch support insoles should have been identical, it seems reasonable to conclude that the reported differences were due to psychological and/or physiological factors associated with colour.

\section{Discussion}

Designers of all aspects of the built environment commonly exploit colour associations and their researched human psychological and physiological responses when developing their products, packaging and marketing materials. Yet manufacturers of medical grade foot orthoses (of the kind commonly prescribed by clinicians, and particularly those manufactured by custom foot orthoses laboratories) appear, to date, to have not exploited this design variable to any great degree. Equally, the research community has seemingly given little thought as to how by simply manipulating the colour of foot orthoses, patient outcomes might be potentially improved upon. 
Somewhat ashamedly, when one looks beyond the typical medical use of foot orthoses and the published foot orthoses research, some insole manufacturers appear to be cognisant of thermal colour associations. A cursory glance at any online retail site will evidence that electronically heated insoles are usually coloured red or orange and that "cooling" gel insoles are frequently coloured blue; this is unlikely to have been a series of serendipitous decisions that all manufacturers of such products have come to independently. Indeed, it seems intuitive to suggest that the warming influence of reds, oranges and yellows might be employed by clinicians within insulating insoles as part of the management of vasospastic disorders such as Raynaud's syndrome and that when patients present with hot, acute inflammatory conditions then red, yellow and orange coloured foot orthoses might be better avoided with blue being the preferred colour for it's "cooling" qualities. However, the counterintuitive results from research regarding colour associations and perceived temperature of physical objects casts some doubt as to the accuracy of these assumptions ${ }^{4}$. Future research should focus on colour and its associated temperature perceptions that are specific to foot orthoses.

Foot orthoses are commonly prescribed to patients experiencing some form of pain (plantar heel pain, osteoarthritic pain, tendinopathy etc). Given the evidence linking the colour red to increased pain perception ${ }^{7}$ and its association with high intensity pain ${ }^{6}$ it would seem reasonable to suggest the avoidance of red coloured foot orthoses for patients presenting with high selfreported pain levels. The association of grey and black with chronic ache type pain leads to a similar conclusion ${ }^{6}$. For patients presenting with pain it would appear that the choice of cooler colours such as green or blue foot orthoses should be more appropriate.

In deciding upon the colour of foot orthoses for the management of pain, it is important that the clinician, if possible, elicits from the patient a description of "their pain" in terms of a colour 6 . It would seem reasonable to avoid any colour described by the patient as being associated with their pain and instead produce foot orthoses that have a colour from the opposite side of the colour wheel.

Research suggests that the colour red may increase the strength and velocity of motor responses ${ }^{10,11}$. When employed within foot orthoses design, red has been associated with increased levels of perceived comfort and to be linked with significant differences in some kinetic measures at the ankle in noninjured athletes ${ }^{13}$. This suggests that while red coloured foot orthoses might be 
better avoided in the injured athlete due to the negative associations with pain (as discussed earlier), perhaps red with its associated stimulant effect ${ }^{8}$ should be the colour of choice when foot orthoses are being provided to uninjured athletes in an attempt to improve their athletic performances. As things stand, there is currently too little evidence to draw definitive conclusions regarding the effect of foot orthoses colour on athletic performance. However, the results from the data published thus far make it worthy of further research investigation.

Given the subjectivity of colour perception and its associations, the subjective nature of pain and the subjectivity of the whole human experience within a biopsychosocial model, perhaps until such time that we have research to provide more definitive answers, simply asking the patient "what colour would you like your foot orthoses to be?" and then providing orthoses in that colour might be the way toward better treatment outcomes with foot orthoses therapy.

\section{Conclusion}

Within the evidence based medicine framework, therapeutic modalities are judged in terms of their ability to bring about positive clinical outcomes. Due to the historical bias toward a biomedical model, most published work in the field of foot orthoses has focused on their direct and indirect mechanical effects upon the musculoskeletal system. However, it cannot be ignored that their employment by clinicians is part of a much bigger biopsychosocial experience of the recipient. While clinicians and foot orthoses manufacturers have perhaps given little thought to the colour of foot orthoses historically, it is an easy variable to manipulate. Clearly the colour of foot orthoses is only one element within the overall design of the product and it is obvious that unless the other design characteristics of the foot orthoses such as their surface geometry and stiffness are well matched to an individual's need, then regardless of the colour, the foot orthoses are unlikely to be clinically efficacious. Nevertheless, manipulation of foot orthoses colour may have some impact on the patient's physiological and psychological functioning. Given its relative ease of implementation, an exploration of the impact that orthoses colour may have on patient outcomes seems ripe for clinical consideration and further research. 


\section{References}

1. Elliot AJ, Maier MA. Color Psychology: Effects of perceiving color on psychological functioning in humans. Annual Review of Psychology. 65:9, 2014

2. Kurt $S$, Osueke KK. The effect of colour on the moods of college students. SAGE Open. 4(1):1, 2014

3. Prokop P, Fancovicova J. Does colour matter? The influence of animal warning coloration on human emotions and willingness to protect them. Animal Conservation. 16:458, 2013

4. Ho HN, Van Doorn GH, Kawabe T, et al. Colour-temperature correspondences: when reactions to thermal stimuli are influenced by colour. PLoS One. 9(3): e91854, 2014

5. Ho HN, Iwai Y, Yoshikawa J, et al. Combining colour and temperature: a blue object is more likely to be judged as warm than a red object. Scientific Reports. 4:5527, 2014

6. Wylde V, Wells V, Dixon S et al. The colour of pain: can patients use colour to describe osteoarthritis pain? Musculoskeletal Care. 12(1):34, 2013

7. Weircioch-Kuzianik K, Babel P. Color hurts. The effect of color on pain perception. Pain Med. 20(10):1955, 2019

8. De Craen AJM, Roos PJ, De Vries AL, et al. Effect of colour of drugs: systematic review of perceived effects of drugs and their effectivenesss. BMJ. 313:1624, 1996

9. Garcia-Rubio MA, Picazo-Tadeo AJ, Gonzalez-Gomez F. Does a red shirt improve sporting performance? Evidence from Spanish football. Applied Economics Letters. 18(11):1001, 2011

10. Elliot AJ, Aarts H. Perception of the colour red enhances the force and velocity of motor output. Emotion. 11(2):445, 2011

11.Dreiskaemper D, Strauss B, Hagemann N. et al. Influence of red color on physical parameters in combat sports. Journal of Sport and Exercise Psychology. 35:44, 2013

12.Lam WK, Kam, K, Qu Y et al. Influence of shoe colour on perceived and actual jumping performance. Footwear Science. 9:sup1, S3, 2017

13. Wang $\mathrm{Y}$, Lam GWK, Cheung $\mathrm{CH}$, et al. Effect of red arch-support insoles on subjective comfort and movement biomechanics in various landing heights. International Journal of Environmental Research and Public Health. 17(7):2476, 2020 